\title{
Nonassociative Weyl star products
}

\author{
V.G. Kupriyanov ${ }^{1}$ and D.V. Vassilevich \\ $C M C C$-Universidade Federal do ABC, \\ Santo André, SP, Brazil \\ E-mail: vladislav.kupriyanov@gmail.com, dvassil@gmail.com
}

ABSTRACT: Deformation quantization is a formal deformation of the algebra of smooth functions on some manifold. In the classical setting, the Poisson bracket serves as an initial conditions, while the associativity allows to proceed to higher orders. Some applications to string theory require deformation in the direction of a quasi-Poisson bracket (that does not satisfy the Jacobi identity). This initial condition is incompatible with associativity, it is quite unclear which restrictions can be imposed on the deformation. We show that for any quasi-Poisson bracket the deformation quantization exists and is essentially unique if one requires (weak) hermiticity and the Weyl condition. We also propose an iterative procedure that allows one to compute the star product up to any desired order.

KEYWORDs: Flux compactifications, Non-Commutative Geometry

ARXiv EPrint: 1506.02329

\footnotetext{
${ }^{1}$ Also at Tomsk State University, Tomsk, Russia.
} 


\section{Contents}

1 Introduction 1

2 Main definitions and the main result 3

3 Associator and Jacobiator 5

4 Resolving the weak hermiticity condition $\quad 6$

5 Explicit formulas at lowest orders $\quad 9$

5.1 Second order 9

5.2 Third order 9

6 Discussion 10

A Important expansion $\quad 12$

\section{Introduction}

Star product are usually understood as deformations of the usual point-wise product on the space of smooth functions on some manifold $M$. The theory of star products was developed mostly in the framework of the deformation quantization approach [1]. The associativity of star products reflects the associativity of compositions of quantum mechanical operators. A milestone result in this direction is the Formality Theorem by Kontsevich [2] that provides a classification of star products on Poisson manifolds and gives a recipe to calculate these products. Some more references and a historic overview may be found in [3, 4].

The interest to star products was boosted by the discovery $[5,6]$ that the coordinates of string endpoints attached to a Dirichlet brane in a background $B$-field do not commute, and therefore, star products are natural to describe correlation functions. It was found a bit later [7-9], that in a non-constant $B$-field, the product should be not only noncommutative, but also nonassociative. More recently, similar effects were discovered in the closed string sector. It was demonstrated [10-12] that the presence of a non-geometric $R$-flux leads to a twist of the Poisson structure and to nonasociativity of corresponding star products. Note, that the mechanism of breaking the associativity is very similar to the one that was found in the background of a magnetic monopole [13]. An extended discussion on the relations between the magnetic field and the nonassociativity may be found in [14]. Despite this renewed interest, the overall situation with twisted Poisson structures and nonassociative star products remains by far less clear than with their associative counterparts.

We are interested in quantizing the quasi-Poisson bracket

$$
\{f, g\}_{Q}=P^{i j}(x) \partial_{i} f \partial_{j} g
$$


without imposing the Jacobi identity on the bivector $P$. It is assumed usually that the failure of $P$ to satisfy the Jacobi identity is proportional to a closed 3 -form, i.e., that one deals with a twisted Poisson structure $[15,16]$. We shall not use this assumption thus keeping $P$ arbitrary. There exists a different definition of quasi-Poisson manifolds [17]. We hope this will not lead to confusion.

Star products admit a formal expansion,

$$
f \star g=f \cdot g+\sum_{r=1}^{\infty}(\mathrm{i} \alpha)^{\mathrm{r}} \mathrm{C}_{\mathrm{r}}(\mathrm{f}, \mathrm{g}),
$$

where $\alpha$ is a deformation parameter, $\mathrm{i} \equiv \sqrt{-1}$, and $C_{r}$ denote some bidifferential operators. These products provide a quantization of the bracket (1.1) if

$$
C_{1}(f, g)-C_{1}(g, f)=2\{f, g\}_{Q} .
$$

Normally [1], higher orders $C_{r}(f, g), r>1$, are restricted by the associativity of star products. The Jacobi identities on $P^{i j}$ follow from the associativity. Any formal Poisson structure defines then a star product up to a "gauge transformation" [2].

In the non-Poisson/nonassociative ${ }^{1}$ case the condition (1.3) can still be imposed, but it is unclear what may play the role of the associativity conditions in restricting the higher orders in (1.2). There are various proposals in the literature. One can use quasi-Hopf twist deformations [23], or exploit the formality theorem and impose the Kontsevich formula for $C_{r}$ as in [24].

In this paper we propose an approach to nonassociative star products that is based on realizations of coordinates $x$ as differential operators $\hat{x}$ and on the Weyl ordering prescription for the functions $\hat{f}(\hat{x})$. This approach is closer to the ideology of quantum mechanics. One may think naively that associativity of the operator composition contradicts nonassociativity of the star product and makes the operator formalism useless in the present context. Constructing a nonassociative Weyl star product seems to be an interesting and challenging problem. We shall be able to construct this product for a completely general bivector $P^{i j}$. Besides, as we shall see below, the Weyl products have considerable computations advantages over other products.

Our method for the construction of nonassociative star products generalizes the one that we proposed earlier [25] for the associative products. The Weyl ordering is a classical tool in the theory of star products [4]. It was used to analyze Fedosov star products on cotangent bundles [26] and gauge theories on noncommutative space [27]. Representation through differential operators also proved very convenient [26], especially with the Liealgebra type noncommutativity [28-35].

This paper is organized as follows. In the next section, we define Weyl, triangular and Hermitian star products and formulate our main result concerning their existence and

\footnotetext{
${ }^{1}$ There is a completely different point of view on the quantization problem related to twisted Poisson structures. Instead of nonassociative deformations of algebras one deals with deformations of gerbes or algebroid stacks, see [18-21]. With some additional restrictions, e.g. when the twisted Poisson structure is prequantizable, one can apply the methods of geometric quantization [22]. We shall not follow any of these approaches here.
} 
uniqueness. In section 3 we derive some properties of associator and jacobiator of star products involving coordinates. Section 4 contains an iterative procedure that allows to compute the star product to any given order. Explicit expressions up to the third order are derived in section 5 . The results are discussed in section 6 , while a long and important expansion can be found in the appendix.

\section{Main definitions and the main result}

Let $A$ be some algebra of functions on $\mathbb{R}^{N}$ with a point-wise product which is closed with respect to derivatives of an arbitrary order. Consistent choices are algebras of smooth functions or algebras of polynomials. Let $P^{i j}$ be some bivector on $\mathbb{R}^{N}$, and let $A[[\alpha]]$ be the algebra of formal power series of $\alpha$ with coefficients in $A$. Star product is any deformation of the point-wise product satisfying (1.2) and (1.3), though we shall consider only those deformations that do not change the unity,

$$
f \star 1=1 \star f=f .
$$

Through the star product one may associate a (formal) differential operator $\hat{f}$ to a function $f$ as

$$
(f \star g)(x)=\hat{f} \triangleright g(x),
$$

where the symbol $\triangleright$ on the right hand side means an action of a differential operator on a function. In particular,

$$
x^{i} \star f=\hat{x}^{i} \triangleright f(x) .
$$

We call the start product the Weyl star product if for any $f$ the operator $\hat{f}$ can be obtained by the Weyl symmetric ordering of operators $\hat{x}^{j}$ corresponding to the coordinates $x^{j}$. If $\tilde{f}(p)$ is a Fourier transform of $f$, then

$$
\hat{f}=\hat{f}(\hat{x})=W(f)=\int \frac{d^{N} p}{(2 \pi)^{N}} \tilde{f}(p) e^{-\mathrm{ip}_{\mathrm{m}} \hat{\mathrm{x}}^{\mathrm{m}}} .
$$

For example, $W\left(x^{i} x^{j}\right)=\frac{1}{2}\left(\hat{x}^{i} \hat{x}^{j}+\hat{x}^{j} \hat{x}^{i}\right)$. Weyl star products satisfy

$$
\left(x^{i_{1}} \ldots x^{i_{n}}\right) \star f=\sum_{P_{n}} \frac{1}{n !} P_{n}\left(x^{i_{1}} \star\left(\cdots \star\left(x^{i_{n}} \star f\right) \ldots\right),\right.
$$

where $P_{n}$ denotes a permutation of $n$ elements. This equation may be even used to define Weyl star products with explicit use of differential operators in the spirit of [29]. For us, the use of operator language will be essential. One can write the formal differential operator $\hat{x}^{i}$, corresponding to the coordinate $x^{i}$, as

$$
\hat{x}^{i}=x^{i}+\sum_{n=1}^{\infty} \Gamma^{i(n)}(\alpha, x)(\mathrm{i} \alpha \partial)^{\mathrm{n}}
$$

where

$$
\Gamma^{i(n)}(\alpha, x)=\Gamma^{i i_{1} \ldots i_{n}}(\alpha, x)
$$


We have shown in [25] that a Weyl star product satisfies the condition (2.1) of stability of unity if and only if

$$
p_{i} p_{i_{1}} \ldots p_{i_{k}} \Gamma^{i i_{1} \ldots i_{k}}=0
$$

where all indices in $\Gamma$ are contracted with commuting variables $p_{i}$. By the construction, eq. (2.5), $\Gamma^{i i_{1} \ldots i_{k}}$ is antisymmetric in the last $n$ indices. Thus, $\Gamma^{i i_{1} \ldots i_{k}}$ may transform under the permutations according to the representations described by the Young tableaus

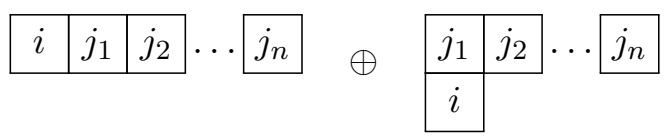

The condition (2.7) kills the first (totally symmetric) component. Following [25] we shall call this condition the tracelessness condition, though, strictly speaking, it is not related to any trace.

A word of warning is in order. The correspondence $f \rightarrow \hat{f}$ is not an algebra representation. Since the star product that we consider here does not need to be associative, in general $\widehat{(f \star g)} \neq \hat{f} \circ \hat{g}$.

The parameter $\alpha$ is a formal expansion parameter that has no numerical value. Therefore, in the formal setting there is no way to compare different powers of $\alpha$, in contrast to the physical situation. For this reason, one may change within certain limits the order assignments for various terms without changing the physical content. Alternatively, one may impose restrictions on the way we organize the formal expansion. One possible restriction is to request a triangular structure of the expansion (1.2): the order of derivatives acting on $f$ or $g$ in any $C_{r}$ may not exceed $r$. Then each $\Gamma$ is expanded in non-negative powers of $\alpha$ :

$$
\Gamma^{i(n)}(\alpha, x)=\sum_{k=0}^{\infty}(\mathrm{i} \alpha)^{\mathrm{k}} \Gamma_{\mathrm{k}}^{\mathrm{i}(\mathrm{n})}(\mathrm{x}) .
$$

We also impose restrictions on the terms with lowest number of derivatives. We assume that the $\Gamma^{i(n)}$ with $n=1$ has no $\alpha$-corrections, i.e.,

$$
\Gamma^{j k}(\alpha, x)=\Gamma_{0}^{j k}(x)
$$

The meaning of this condition will be discussed later, see section 6 . The triangular star products satisfying (2.10) will be called strictly triangular.

Further conditions refer to the properties of star product related with complex conjugation. A star product is called Hermitian if

$$
(g \star f)^{*}=f^{*} \star g^{*} .
$$

We shall use a weaker condition, that we shall call weak Hermiticity:

$$
\left(x^{j} \star f\right)^{*}=f^{*} \star x^{j}
$$

for all $x^{j}$. 
We are ready now to formulate the main result of this paper:

Proposition 1 For any bivector field $P^{i j}$ there is unique weakly Hermitian strictly triangular Weyl star product satisfying the stability of unity condition.

Moreover, we shall present recursion relations that allow to compute this star product to any given order.

\section{Associator and Jacobiator}

In this section we study algebraic properties of weakly Hermitian Weyl star products. The associator $A(f, g, h)$ and Jacobiator $J(f, g, h)$ are defined by the formulas

$$
A(f, g, h)=f \star(g \star h)-(f \star g) \star h,
$$

and

$$
J(f, g, h)=\frac{1}{6}\left\{\left[f,[g, h]_{\star}\right]_{\star}+\left[h,[f, g]_{\star}\right]_{\star}+\left[g,[h, f]_{\star}\right]_{\star}\right\},
$$

where $[f, g]_{\star}$ stands for the star commutator. By the definition, the Jacobiator is antisymmetric over all arguments, while the associator is not necessarily so. One finds the relation

$$
J(f, g, h)=\frac{1}{6}\{A(f, g, h)-A(f, h, g)+A(h, f, g)-A(h, g, f)+A(g, h, f)-A(g, f, h)\} .
$$

A nonassociative star product may have a vanishing Jacobiator.

For the Weyl star product, one has by (2.4)

$$
\left(x^{i} x^{j}\right) \star x^{k}=\frac{1}{2}\left(x^{i} \star\left(x^{j} \star x^{k}\right)+x^{j} \star\left(x^{i} \star x^{k}\right)\right) .
$$

On the other hand the stability of the unity,

$$
\frac{1}{2}\left(x^{i} \star x^{j}+x^{j} \star x^{i}\right)=x^{i} x^{j},
$$

implies

$$
\left(x^{i} \star x^{j}\right) \star x^{k}+\left(x^{j} \star x^{i}\right) \star x^{k}=x^{i} \star\left(x^{j} \star x^{k}\right)+x^{j} \star\left(x^{i} \star x^{k}\right) .
$$

Which means that

$$
A\left(x^{i}, x^{j}, x^{k}\right)+A\left(x^{j}, x^{i}, x^{k}\right)=0,
$$

that is, the associator of coordinate functions $x^{i}, x^{j}$ and $x^{k}$ is antisymmetric in first two arguments.

If the Weyl star product is weak-Hermitian, then considering complex conjugate of the equation (3.5) and using (2.12) we obtain

$$
x^{k} \star\left(x^{j} \star x^{i}\right)+x^{k} \star\left(x^{i} \star x^{j}\right)=\left(x^{k} \star x^{j}\right) \star x^{i}+\left(x^{k} \star x^{i}\right) \star x^{j} .
$$

That is, the associator of coordinate functions $x^{k}, x^{j}$ and $x^{i}$ is antisymmetric in the last two arguments,

$$
A\left(x^{k}, x^{j}, x^{i}\right)+A\left(x^{k}, x^{i}, x^{j}\right)=0 .
$$


Using (3.6) and (3.8) in (3.3) we find

$$
J\left(x^{i}, x^{j}, x^{k}\right)=A\left(x^{i}, x^{j}, x^{k}\right) .
$$

For the weak-Hermitian Weyl star product the associator $A\left(x^{i}, x^{j}, x^{k}\right)$ is antisymmetric in all arguments and is equal to the Jacobiator $J\left(x^{i}, x^{j}, x^{k}\right)$. Antisymmetry of the associator plays an important role in the construction of [36].

\section{Resolving the weak hermiticity condition}

In this section we shall rewrite the weak hermiticity condition as a system of coupled algebraic equations and develop an iterative procedure that always gives a unique solution to that equations. By eq. (2.3), for any Weyl star product this condition is equivalent to the following equation

$$
\left(\int \frac{d^{N} p}{(2 \pi)^{N}} \tilde{f}(p) e^{-\mathrm{ip}_{\mathrm{j}} \hat{\mathrm{x}}^{\mathrm{j}}} \triangleright x^{i}\right)^{*}=\int \frac{d^{N} p}{(2 \pi)^{N}} \tilde{f}^{*}(p) \hat{x}^{i} \triangleright e^{\mathrm{i} \mathrm{p}_{\mathrm{j}} \mathrm{x}^{\mathrm{j}}},
$$

which holds true if and only if

$$
e^{-i p_{\mathrm{j}} \hat{\mathrm{x}}^{\mathrm{j}}} \triangleright x^{i}=\left(\hat{x}^{i} \triangleright e^{\mathrm{i} \mathrm{p}_{\mathrm{j}} \mathrm{x}^{\mathrm{j}}}\right)^{*}
$$

The right-hand side of this equation is

$$
\left[\left(x^{i}+\sum_{n=1}^{\infty} \Gamma^{i(n)}(\alpha, x)(\mathrm{i} \alpha \partial)^{\mathrm{n}}\right) \mathrm{e}^{\mathrm{i} \mathrm{p}_{\mathrm{j}} \mathrm{x}^{\mathrm{j}}}\right]^{*}=\mathrm{e}^{-\mathrm{i} \mathrm{p}_{\mathrm{j}} \mathrm{x}^{\mathrm{j}}}\left(\mathrm{x}^{\mathrm{i}}+\sum_{\mathrm{n}=1}^{\infty} \alpha^{\mathrm{n}}(-1)^{\mathrm{n}} \sum_{\mathrm{k}=0}^{\mathrm{n}-1} \mathrm{i}^{\mathrm{k}} \Gamma_{\mathrm{k}}^{\mathrm{i}(\mathrm{n}-\mathrm{k}) *}(\mathrm{x})(\mathrm{p})^{\mathrm{n}-\mathrm{k}}\right) .
$$

On the left hand side of (4.1) one needs to expand the operator $\exp \left[-\mathrm{ip}_{\mathrm{j}} \hat{\mathrm{x}}^{\mathrm{j}}\right]$ to a given order of $\alpha$. To this end we shall use the Duhamel formula

$$
e^{A+B}=e^{A}+\int_{0}^{1} e^{(A+B) s} B e^{(1-s) A} d s .
$$

Here $A+B=-\mathrm{ip}_{\mathrm{i}} \hat{\mathrm{x}}^{\mathrm{i}}, A=-\mathrm{ip}_{\mathrm{i}} \mathrm{x}^{\mathrm{i}}$ and $B=-\mathrm{ip}_{\mathrm{i}}\left(\hat{\mathrm{x}}^{\mathrm{i}}-\mathrm{x}^{\mathrm{i}}\right)$. By using these rules, one finds,

$$
\begin{aligned}
e^{A+B}=e^{A}( & 1+B+\frac{1}{2}[B, A]+\frac{1}{2} B^{2} \\
& +\frac{1}{6}[[B, A], A]+\frac{1}{3}[B, A] B+\frac{1}{6} B[B, A]+\frac{1}{6} B^{3} \\
& +\frac{1}{24}[[[B, A], A], A]+\frac{1}{8}[[B, A], A] B+\frac{1}{8}[B, A]^{2} \\
& +\frac{1}{24} B[[B, A], A]+\frac{1}{8}[B, A] B^{2}+\frac{1}{12} B[B, A] B \\
& \left.+\frac{1}{24} B^{2}[B, A]+\frac{1}{24} B^{4}+\ldots\right) .
\end{aligned}
$$

Let us separate on the right hand side of (4.4) the terms that are linear in $B$. These are repeated commutators of the form $[\ldots[B, A], \ldots, A]$. Denote $B_{m}=-\mathrm{ip}_{\mathrm{j}} \Gamma^{\mathrm{j}(\mathrm{m})}(\alpha, \mathrm{x})(\mathrm{i} \alpha \partial)^{\mathrm{m}}$. 
Then nonvanishing contributions may be calculated taking into account the condition (2.7):

$$
\begin{aligned}
& {\left[B_{2}, A\right] \triangleright x^{i} }=2(\mathrm{i} \alpha)^{2}\left(-\mathrm{ip}_{\mathrm{j}}\right)\left(-\mathrm{ip}_{\mathrm{i}_{1}}\right) \Gamma^{\mathrm{ji}_{1} \mathrm{i}_{2} \mathrm{i}}, \\
& {\left[\left[B_{3}, A\right], A\right] } \triangleright x^{i}=3 !(\mathrm{i} \alpha)^{3}\left(-\mathrm{ip}_{\mathrm{j}}\right)\left(-\mathrm{ip}_{\mathrm{i}_{1}}\right)\left(-\mathrm{ip}_{\mathrm{i}_{2}}\right) \Gamma^{\mathrm{ji}_{1} \mathrm{i}_{2} \mathrm{i}}, \\
& {\left[\left[\left[B_{4}, A\right], A\right], A\right] \triangleright x^{i}=4 !(\mathrm{i} \alpha)^{4}\left(-\mathrm{ip}_{\mathrm{j}}\right)\left(-\mathrm{ip}_{\mathrm{i}_{1}}\right)\left(-\mathrm{ip}_{\mathrm{i}_{2}}\right)\left(-\mathrm{ip}_{\mathrm{i}_{3}}\right) \Gamma^{\mathrm{ji}_{1} \mathrm{i}_{2} \mathrm{i}_{3} \mathrm{i}} . }
\end{aligned}
$$

That is,

$$
\left[\ldots[B, \underbrace{A] \ldots, A}_{m-1}] \triangleright x^{i}=m !(\mathrm{i} \alpha)^{\mathrm{m}} \Gamma^{(\mathrm{m}) \mathrm{i}}(\alpha, \mathrm{x})(-\mathrm{ip})^{\mathrm{m}} .\right.
$$

Finally we conclude that the contribution of the repeated commutators to the l.h.s. of (4.1) is given by

$$
\begin{aligned}
e^{-\mathrm{ip}_{\mathrm{j}} \mathrm{x}^{\mathrm{j}}} \sum_{m=2}^{\infty} \frac{1}{(m) !}\left[\ldots[B, \underbrace{A] \ldots, A}_{m-1}] \triangleright x^{i}\right. & =e^{-\mathrm{ip}_{\mathrm{j}} \mathrm{x}^{\mathrm{j}}} \sum_{m=2}^{\infty} \alpha^{m}\left(\sum_{k=0}^{\infty}(\mathrm{i} \alpha)^{\mathrm{k}} \Gamma_{\mathrm{k}}^{(\mathrm{m}) \mathrm{i}}(\mathrm{x})\right)(\mathrm{p})^{\mathrm{m}} \\
& =e^{-\mathrm{ip}_{\mathrm{j}} \mathrm{x}^{\mathrm{j}}} \sum_{n=2}^{\infty} \alpha^{n} \sum_{k=0}^{n-1} \mathrm{i}^{\mathrm{k}} \Gamma_{\mathrm{k}}^{(\mathrm{n}-\mathrm{k}) \mathrm{i}}(\mathrm{x})(\mathrm{p})^{\mathrm{n}-\mathrm{k}}
\end{aligned}
$$

This allows us to rewrite the equation (4.1) as

$$
\begin{aligned}
\sum_{n=2}^{\infty} \alpha^{n} & \sum_{k=0}^{n-1} \mathrm{i}^{\mathrm{k}}\left((-1)^{\mathrm{n}} \Gamma_{\mathrm{k}}^{\mathrm{i}(\mathrm{n}-\mathrm{k}) *}-\Gamma_{\mathrm{k}}^{(\mathrm{n}-\mathrm{k}) \mathrm{i}}\right)(\mathrm{p})^{(\mathrm{n}-\mathrm{k})}= \\
& \left(\frac{1}{2} B^{2}+\frac{1}{3}[B, A] B+\frac{1}{6} B[B, A]+\frac{1}{6} B^{3}+\frac{1}{8}[[B, A], A] B+\frac{1}{8}[B, A]^{2}\right. \\
& +\frac{1}{24} B[[B, A], A]+\frac{1}{8}[B, A] B^{2}+\frac{1}{12} B[B, A] B \\
& \left.+\frac{1}{24} B^{2}[B, A]+\frac{1}{24} B^{4}+\ldots\right) \triangleright x^{i} .
\end{aligned}
$$

Let us also expand the right hand side of equation above in a power series of $\alpha$ and $p$

$$
\sum_{n=2}^{\infty} \alpha^{n} \sum_{k=0}^{n-1} \mathrm{i}^{\mathrm{k}} \mathrm{F}^{\mathrm{i}(\mathrm{n}-\mathrm{k})}(\mathrm{p})^{(\mathrm{n}-\mathrm{k})} .
$$

By comparing the terms with the same power of $\alpha$ and $p$ in the right and left hand sides of (4.7) we arrive at

$$
\left(\Gamma_{k}^{i(m) *}-(-1)^{k+m} \Gamma_{k}^{(m) i}\right)(p)^{m}=(-1)^{k+m} F_{k}^{i(m)}(p)^{m}, \quad k+m=n .
$$

With the help of the condition (2.7) and by using the symmetry properties of $\Gamma$, one derives that

$$
\Gamma_{k}^{(m) i}(p)^{m}=-\frac{1}{m} \Gamma_{k}^{i(m)}(p)^{m} .
$$

This yields for $m \geq 2$

$$
\begin{aligned}
\operatorname{Re} \Gamma_{k}^{i(m)}(p)^{m} & =\frac{m}{1+(-1)^{m+k} m} \operatorname{Re} F_{k}^{i(m)}(p)^{m} \\
\operatorname{Im} \Gamma_{k}^{i(m)}(p)^{m} & =\frac{m}{1-(-1)^{m+k} m} \operatorname{Im} F_{k}^{i(m)}(p)^{m} .
\end{aligned}
$$


Both sides of both equations (4.11) correspond to the same order $\alpha^{n}=\alpha^{m+k}$. However, since all terms on the right hand side of eq. (4.7) are at least quadratic in $B$ (and, consequently, also in $\Gamma$ ) the tensors $F_{k}^{i(m)}$ are defined through the $\Gamma$ 's in the order strictly less than $n$. In other words, if one knows all $\Gamma$ at the orders less than $n$, one can calculate $F_{k}^{i(m)}$ for $m+k=n$.

To show that the right hand sides of (4.11) always define uniquely the left hand sides, it is enough to show that $F_{k}^{i(m)}$ is always traceless. Let us pick up a term in (4.7). By the arguments similar to that around eq. (4.5), a non-vanishing contribution of such a term should read

$$
D_{n-k-l}(\Gamma, \partial, p) \Gamma_{k}^{(l) i}(-\mathrm{ip})^{1}, \quad \mathrm{k}+\mathrm{l}<\mathrm{n},
$$

where we have separated explicitly the rightmost $\Gamma . D_{n-k-l}(\Gamma, \partial, p)$ is some differential operator. Since all $\Gamma$ 's are supposed to be traceless, contracting (4.12) with $p_{i}$ yields zero, which means that $F_{k}^{i(m)}$ is traceless as well.

We see, that indeed the equations allow us to define all terms $\Gamma_{k}^{i(m)}$ with $m+k=n$ if the terms with $m+k<n$ are known. This procedure works for $m>1$ only, so that the case $m=1$ has to be treated separately. Due to (2.9) one has to analyze $\Gamma_{0}^{i j}$ only. The symmetric part vanishes because of (2.7),

$$
\Gamma_{0}^{j_{1} i}+\Gamma_{0}^{i j_{1}}=0
$$

The antisymmetric part is fixed by (1.3). Indeed, by taking $f=x^{i}, g=x^{j}$ we immediately obtain

$$
\Gamma^{i j}(\alpha, x)=\Gamma_{0}^{i j}(x)=P^{i j}(x)
$$

Next we note that the right hand side of (4.9) does not contain imaginary coefficients. All imaginary units appear in combinations with $\alpha$ or with $p$, but the corresponding powers of i are explicitly isolated in (4.8). Therefor, if all $\Gamma_{k}^{i(m)}$ with $m+k<n$ are real, then $F_{k}^{i(m)}$ with $k+m=n$ are also real, and by (4.11) all $\Gamma_{k}^{i(m)}$ with $k+m=n$ are real as well. Since the lowest order $\Gamma_{0}^{i j}$ is real by eq. (4.14), all $\Gamma_{k}^{i(m)}$ are real for all $k$ and $m$. Consequently, the only iteration relation that we need is

$$
\Gamma_{k}^{i(m)}(p)^{m}=\frac{m}{1+(-1)^{m+k} m} F_{k}^{i(m)}(p)^{m} .
$$

An alternative way to obtain the equations (4.9) is to consider the weak hermiticity conditions taking the function $f$ in the form of monomials,

$$
s_{i} x^{i} \star\left(q_{j_{1}} \ldots q_{j_{n}} x^{j_{1}} \ldots x^{j_{n}}\right)=\left[\left(q_{j_{1}} \ldots q_{j_{n}} x^{j_{1}} \ldots x^{j_{n}}\right) \star x^{i} s_{i}\right]^{*} .
$$

The interested reader may supply necessary technical details. 


\section{$5 \quad$ Explicit formulas at lowest orders}

\subsection{Second order}

Let us use the expansion (A.1) to write down the weak hermiticity condition (4.1) to the second order in $\alpha$ :

$$
\begin{gathered}
e^{-\mathrm{i} \mathrm{p}_{\mathrm{j}} \mathrm{x}^{\mathrm{j}}}\left[x^{i}+\alpha p_{j_{1}} \Gamma_{0}^{j_{1} i}+\alpha^{2} p_{j_{1}} p_{j_{2}} \Gamma_{0}^{j_{1} j_{2} i}+\frac{\alpha^{2}}{2} p_{j_{1}} p_{j_{2}} \Gamma_{0}^{j_{1} l_{1}} \partial_{l_{1}} \Gamma_{0}^{j_{2} i}\right]= \\
e^{-\mathrm{i} \mathrm{p}_{\mathrm{j}} \mathrm{x}^{\mathrm{j}}}\left[x^{i}-\alpha p_{j_{1}} \Gamma_{0}^{i j_{1}}+\alpha^{2} p_{j_{1}} p_{j_{2}} \Gamma_{0}^{i j_{1} j_{2}}\right]+o\left(\alpha^{2}\right) .
\end{gathered}
$$

Due to (4.13), there is no new restriction at the order of $\alpha^{1}$, though at the second order we obtain the algebraic equation on $\Gamma_{0}^{i j k}$ in terms of $\Gamma_{0}^{i j}$ :

$$
\left(\Gamma_{0}^{i j_{1} j_{2}}-\Gamma_{0}^{j_{1} j_{2} i}\right) p_{j_{1}} p_{j_{2}}=\frac{1}{2} \Gamma_{0}^{j_{1} l_{1}} \partial_{l_{1}} \Gamma_{0}^{j_{2} i} p_{j_{1}} p_{j_{2}},
$$

which yields

$$
\Gamma_{0}^{i j k}=\frac{1}{6}\left(P^{k l} \partial_{l} P^{j i}+P^{j l} \partial_{l} P^{k i}\right) .
$$

The star product is then recovered by using the relation $f \star g=\hat{f} \triangleright g$ and the representation (2.3) for the Weyl ordered operator $\hat{f}$ together with the expansion (A.1). In short, one can take (A.1) and replace there all partial derivatives by derivatives of $g$ and all momenta $\left(-\mathrm{iq}_{\mathrm{j}}\right)$ by partial derivatives of $f, \partial_{j} f$. Taking into account (4.14), (5.3) and the decomposition in the appendix the expression for the star product up to the third order reads

$$
\begin{aligned}
(f \star g)(x)= & f \cdot g+\mathrm{i} \alpha \partial_{\mathrm{i}} \mathrm{fP}^{\mathrm{ij}} \partial_{\mathrm{j}} \mathrm{g} \\
& -\frac{\alpha^{2}}{2} P^{i j} P^{k l} \partial_{i} \partial_{k} f \partial_{j} \partial_{l} g-\frac{\alpha^{2}}{3} P^{i j} \partial_{j} P^{k l}\left(\partial_{i} \partial_{k} f \partial_{l} g-\partial_{k} f \partial_{i} \partial_{l} g\right)+o\left(\alpha^{2}\right) .
\end{aligned}
$$

\subsection{Third order}

In the third order we need algebraic equations on two functions $\Gamma_{0}^{i j k l}$ and $\Gamma_{1}^{i j k}$. The first one reads

$$
\left(\Gamma_{0}^{i j k l}+\Gamma_{0}^{j k l i}\right) q_{j} q_{k} q_{l}=-F_{0}^{i j k l} q_{j} q_{k} q_{l},
$$

where

$$
F_{0}^{i j k l}=\frac{1}{6} P^{j m} \partial_{m} P^{k n} \partial_{n} P^{l i}+\frac{1}{6} P^{j m} P^{k n} \partial_{m} \partial_{n} P^{l i}+\frac{2}{3} \Gamma_{0}^{j k m} \partial_{m} P^{l i}+\frac{1}{3} P^{j m} \partial_{m} \Gamma_{0}^{k l i} .
$$

The second algebraic equation is

$$
\left(\Gamma_{1}^{i j k}+\Gamma_{1}^{j k i}\right) q_{j} q_{k}=-F_{1}^{i j k} q_{j} q_{k},
$$

where

$$
F_{1}^{i j k}=\frac{1}{2} \Gamma_{0}^{k m n} \partial_{m} \partial_{n} P^{j i} .
$$

Both equations can be solved easily by using the relation (4.15). One obtains

$$
\Gamma_{0}^{i j k l}=\frac{1}{18}\left(P^{j m} P^{k n} \partial_{m} \partial_{n} P^{i l}+P^{l m} P^{k n} \partial_{m} \partial_{n} P^{i j}+P^{j m} P^{l n} \partial_{m} \partial_{n} P^{i k}\right),
$$


and

$$
\Gamma_{1}^{i j k}=\frac{1}{6}\left(P^{l m} \partial_{l} P^{k n} \partial_{m} \partial_{n} P^{i j}+P^{l m} \partial_{l} P^{j n} \partial_{m} \partial_{n} P^{i k}\right) .
$$

The star product can be obtained in the manner as we have already described above eq. (5.4). Let us write the 3rd order part of it as

$$
f \star_{3} g=-\mathrm{i} \alpha^{3} \sum \mathrm{L}_{\mathrm{m}, \mathrm{n}}(\mathrm{f}, \mathrm{g}),
$$

where each term in the sum has $m$ derivatives acting on $f$ and $n$ derivatives acting on $g$. Since, $\Gamma_{2}^{i j}=0$,

$$
L_{1,1}(f, g)=0
$$

Further terms read

$$
\begin{aligned}
L_{1,2}(f, g) & =\partial_{i} f \Gamma_{2}^{i j k} \partial_{j} \partial_{k} g=\frac{1}{3} \partial_{i} f P^{l m} \partial_{l} P^{k n} \partial_{m} \partial_{n} P^{i j} \partial_{j} \partial_{k} g, \\
L_{1,3}(f, g) & =\partial_{i} f \Gamma_{1}^{i j k l} \partial_{j} \partial_{k} \partial_{l} g=\frac{1}{6} \partial_{i} f P^{j m} P^{k n} \partial_{m} \partial_{n} P^{i l} \partial_{j} \partial_{k} \partial_{l} g, \\
L_{2,3}(f, g) & =\frac{1}{3} \partial_{i} \partial_{j} f P^{i k} P^{l n} \partial_{l} P^{j m} \partial_{k} \partial_{m} \partial_{n} g, \\
L_{2,2}(f, g) & =\partial_{i} \partial_{j} f\left[\frac{3}{2} \Gamma_{0}^{i j k l}+\frac{1}{2} P^{i m} \partial_{m} \Gamma_{0}^{j k l}+\Gamma_{0}^{i k m} \partial_{m} P^{j l}\right] \partial_{k} \partial_{l} g \\
& =\frac{1}{6} P^{n k} \partial_{n} P^{j m} \partial_{m} P^{i l}\left(\partial_{i} \partial_{j} f \partial_{k} \partial_{l} g-\partial_{i} \partial_{j} g \partial_{k} \partial_{l} f\right) .
\end{aligned}
$$

One can check at this order that

$$
L_{m, n}(f, g)=-L_{n, m}(g, f) \text {. }
$$

By collecting all the terms above, we obtain

$$
\begin{aligned}
f \star_{3} g=-\mathrm{i} \alpha^{3}[ & {\left[\frac{1}{3} P^{n l} \partial_{l} P^{m k} \partial_{n} \partial_{m} P^{i j}\left(\partial_{i} f \partial_{j} \partial_{k} g-\partial_{i} g \partial_{j} \partial_{k} f\right)\right.} \\
& +\frac{1}{6} P^{n k} \partial_{n} P^{j m} \partial_{m} P^{i l}\left(\partial_{i} \partial_{j} f \partial_{k} \partial_{l} g-\partial_{i} \partial_{j} g \partial_{k} \partial_{l} f\right) \\
& +\frac{1}{3} P^{l n} \partial_{l} P^{j m} P^{i k}\left(\partial_{i} \partial_{j} f \partial_{k} \partial_{n} \partial_{m} g-\partial_{i} \partial_{j} g \partial_{k} \partial_{n} \partial_{m} f\right) \\
& +\frac{1}{6} P^{j l} P^{i m} P^{k n} \partial_{i} \partial_{j} \partial_{k} f \partial_{l} \partial_{n} \partial_{m} g \\
& \left.+\frac{1}{6} P^{n k} P^{m l} \partial_{n} \partial_{m} P^{i j}\left(\partial_{i} f \partial_{j} \partial_{k} \partial_{l} g-\partial_{i} g \partial_{j} \partial_{k} \partial_{l} f\right)\right] .
\end{aligned}
$$

We notice that the calculation of this star product is relatively easy.

\section{Discussion}

In this paper we have demonstrated that for any bivector field, there exists a unique Weyl weakly Hermitian strictly triangular star product. Our proof is constructive: the coefficients $\Gamma_{k}^{i(m)}$ are defined by (4.15) through the coefficients $F_{k}^{i(m)}$, which are defined through 
the expansion (4.8) by lower order $\Gamma_{k}^{i(m)}$. By repeating these steps one can express any $\Gamma_{k}^{i(m)}$ through $\Gamma^{i j}$, which appears to be our bivector $P^{i j}$, see (4.14). One may allow $\Gamma^{i j}$ to have higher order contributions, but the corresponding ambiguity is rather non-interesting. Physically, one may say that the higher order corrections are absorbed in renormalization of the bivector $P^{i j}$. The renormalization is of course possible since $P^{i j}$ is arbitrary. This is in contrast to associative Weyl star products. While the initial bivector in the associative case is a Poisson bivector, the corrections are non-Poisson [25, 37]. Consequently, the higher order corrections are non-removable and important. ${ }^{2}$ Therefore, just imposing the Jacobi identity on $P^{i j}$ does not make our star product associative, one still needs additional corrections terms in $\Gamma^{i j}$.

Let us study which of our requirements are satisfied by the nonassociative star products appearing in the literature $[7-9,14,23,24]$. The triangularity condition is automatic if one likes to express the star product through the bivector $P^{i j}$ and its derivatives only. ${ }^{3}$ Then, one does not even need to write the deformation parameter explicitly since the order in the perturbative expansion may be counted by the order of $P^{i j}$. Since $P^{i j}$ has two upper indices, in $C_{r}(f, g)$ one has $2 r$ upper indices to be contracted with lower indices of the derivatives acting on $P, f$ and $g$. Since the terms containing $P^{i j} \partial_{i} \partial_{j} f$ vanish, one has at most $r$ derivatives acting on each of the functions. This means that the star product is triangular, cf. [7, 8, 14, 23, 24]. There is no fundamental reason to expect a start product being strictly triangular, though this property is important to show the uniqueness and easy to achieve by a renormalization of $P^{i j}$ (see above). The products of $[7,8,14,23,24]$ are strictly triangular (at least to the order to which these products are written down explicitly), while the product of [9] is not.

The hermiticity condition (2.11) is very natural, as well as its weaker version (2.12). Such conditions are usually imposed in all quantization schemes. Therefore, no wonder that all products $[7-9,14,23,24]$ appear to be hermitian, at least to the order to which they are known. On the contrary, the Weyl ordering reflects a very particular quantization prescription. To obtain a unique star products one should of course fix the quantization prescription, but this is also the main reason why our star product may differ from other star products.

The papers [7, 8] contain the whole 2 nd order and some terms in the 3rd order of star product following from the open strings/D-branes calculations. All these terms are in perfect agreement with our expressions (5.4) and (5.15). Probably, this is not accidental but postpone the complete analysis to a future work.

Our product differs from the one used in the context of the $R$-flux string models [14, $23,24]$, and also from the one computed by the Kontsevich formulas [2]. (There is some degree of confusion in the literature regarding the form of Kontsevich product at the second order of deformation parameter. See [29] for explicit calculations.) All these products are not Weyl. Since our method works for a completely general bivector $P^{i j}$ we do not have

\footnotetext{
${ }^{2}$ According to Dito [38] these corrections correspond to Kontsevich diagrams with wheels.

${ }^{3}$ Of all papers cited above just a single one [9] uses an additional structure (the Born-Infeld measure) apart of the bivector to construct the star product. However, even the star product of ref. [9] can be made triangular by assigning properly the orders of deformation parameter.
} 
to impose any restrictions of the $R$-fluxes, distributions of magnetic charge, etc. One may easily check that our star product reproduces the same commutators and the same jacobiators involving the coordinates as that of refs. [14, 23, 24], though other quantities may, of course, differ.

Associative star products may be represented through path integrals of Poisson sigma model [39] on a disc [40] or on a finite cylinder [41]. It is an open problem to verify whether our nonassociative star product also admits a path integral interpretation.

We have good grounds to believe that the full hermiticity property (2.11) holds for our star product. We were able to check this at some examples, but a complete proof is still missing.

\section{Acknowledgments}

We are grateful to Jim Stasheff for his detailed comments on the previous version of this manuscript. This work was supported in part by CNPq, projects 443436/2014-2 (V.K.), 306208/2013-0 (D.V.) and 456698/2014-0 (D.V.), and FAPESP, projects 2014/03578-6 (V.K.) and 2012/00333-7 (D.V.).

\section{A Important expansion}

Here using the form (2.5) of the operator $\hat{x}^{i}$ and the decomposition (4.4) we obtain the expression for the operator $\exp \left(-\mathrm{iq}_{\mathrm{i}} \hat{\mathrm{x}}^{\mathrm{i}}\right)$ up to the third order in $\alpha$ :

$$
\begin{aligned}
& e^{-\mathrm{iq}_{\mathrm{i}} \hat{\mathrm{x}}^{\mathrm{i}}}=e^{-\mathrm{i} \mathrm{q}_{\mathrm{i}} \mathrm{x}^{\mathrm{i}}}\left(1+\mathrm{i} \alpha\left(-\mathrm{iq} \mathrm{q}_{\mathrm{i}}\right) \Gamma_{0}^{\mathrm{ij}} \partial_{\mathrm{j}}\right. \\
& +\alpha^{2}\left\{\left(-\mathrm{i} \mathrm{q}_{\mathrm{i}}\right) \Gamma_{0}^{\mathrm{ijk}} \partial_{\mathrm{j}} \partial_{\mathrm{k}}+(-\mathrm{iq} \mathrm{i})(-\mathrm{iq} \mathrm{j})\left[\Gamma_{0}^{\mathrm{ijk}}+\frac{1}{2} \Gamma_{0}^{\mathrm{im}} \partial_{\mathrm{m}} \Gamma_{0}^{\mathrm{jk}}\right] \partial_{\mathrm{k}}\right. \\
& \left.+\left(-\mathrm{iq}_{\mathrm{i}}\right)\left(-\mathrm{iq} \mathrm{q}_{\mathrm{j}}\right) \frac{1}{2} \Gamma_{0}^{\mathrm{ik}} \Gamma_{0}^{\mathrm{jl}} \partial_{\mathrm{k}} \partial_{\mathrm{l}}\right\} \\
& -\mathrm{i} \alpha^{3}\left\{\left(-\mathrm{i} \mathrm{q}_{\mathrm{i}}\right) \Gamma_{1}^{\mathrm{ijk}} \partial_{\mathrm{j}} \partial_{\mathrm{k}}+\left(-\mathrm{iq} \mathrm{q}_{\mathrm{i}}\right) \Gamma_{0}^{\mathrm{ijkl}} \partial_{\mathrm{j}} \partial_{\mathrm{k}} \partial_{\mathrm{l}}\right. \\
& +\left(-i q_{i}\right)\left(-i q_{j}\right)\left[\Gamma_{1}^{\mathrm{ijk}}+\frac{1}{2} \Gamma_{0}^{\mathrm{imn}} \partial_{\mathrm{m}} \partial_{\mathrm{n}} \Gamma_{0}^{\mathrm{jk}}\right] \partial_{\mathrm{k}}+\left(-\mathrm{iq}_{\mathrm{i}}\right)(-\mathrm{iq} \mathrm{j}) \Gamma_{0}^{\mathrm{ik}} \Gamma_{0}^{\mathrm{j} \mathrm{lm}} \partial_{\mathrm{k}} \partial_{\mathrm{l}} \partial_{\mathrm{m}} \\
& +\left(-\mathrm{iq}_{\mathrm{i}}\right)\left(-\mathrm{iq} \mathrm{q}_{\mathrm{j}}\right)\left[\frac{3}{2} \Gamma_{0}^{\mathrm{ijkl}}+\frac{1}{2} \Gamma_{0}^{\mathrm{im}} \partial_{\mathrm{m}} \Gamma_{0}^{\mathrm{jkl}}+\Gamma_{0}^{\mathrm{ikm}} \partial_{\mathrm{m}} \Gamma_{0}^{\mathrm{jl}}\right] \partial_{\mathrm{k}} \partial_{\mathrm{l}} \\
& +\left(-\mathrm{iq} \mathrm{i}_{\mathrm{i}}\right)(-\mathrm{iq} \mathrm{j})(-\mathrm{iq} \mathrm{k})\left[\Gamma_{0}^{\mathrm{ijkl}}+\frac{1}{6} \Gamma_{0}^{\mathrm{im}} \partial_{\mathrm{m}} \Gamma_{0}^{\mathrm{jn}} \partial_{\mathrm{n}} \Gamma_{0}^{\mathrm{kl}}+\frac{1}{6} \Gamma_{0}^{\mathrm{im}} \Gamma_{0}^{\mathrm{jn}} \partial_{\mathrm{m}} \partial_{\mathrm{n}} \Gamma_{0}^{\mathrm{kl}}\right. \\
& \left.+\frac{2}{3} \Gamma_{0}^{i j m} \partial_{m} \Gamma_{0}^{k l}+\frac{1}{3} \Gamma_{0}^{i m} \partial_{m} \Gamma_{0}^{j k l}\right] \partial_{l} \\
& +\left(-i q_{i}\right)\left(-i q_{j}\right)\left(-i q_{k}\right)\left[\frac{1}{2} \Gamma_{0}^{\mathrm{im}} \Gamma_{0}^{\mathrm{jn}} \partial_{\mathrm{n}} \Gamma_{0}^{\mathrm{kl}}+\Gamma_{0}^{\mathrm{ijm}} \Gamma_{0}^{\mathrm{kl}}\right] \partial_{\mathrm{l}} \partial_{\mathrm{m}} \\
& \left.+\left(-\mathrm{iq}_{\mathrm{i}}\right)(-\mathrm{iq} \mathrm{j})\left(-\mathrm{i} \mathrm{q}_{\mathrm{k}}\right) \frac{1}{6} \Gamma_{0}^{\mathrm{im}} \Gamma_{0}^{\mathrm{jn}} \Gamma_{0}^{\mathrm{kl}} \partial_{\mathrm{l}} \partial_{\mathrm{m}} \partial_{\mathrm{n}}\right\}+\mathrm{o}\left(\alpha^{3}\right) .
\end{aligned}
$$


Open Access. This article is distributed under the terms of the Creative Commons Attribution License (CC-BY 4.0), which permits any use, distribution and reproduction in any medium, provided the original author(s) and source are credited.

\section{References}

[1] F. Bayen, M. Flato, C. Fronsdal, A. Lichnerowicz and D. Sternheimer, Deformation theory and quantization. I. Deformations of symplectic structures, Annals Phys. 111 (1978) 61 [INSPIRE].

[2] M. Kontsevich, Deformation quantization of Poisson manifolds, Lett. Math. Phys. 66 (2003) 157 [q-alg/9709040] [INSPIRE].

[3] G. Dito and D. Sternheimer, Deformation quantization: genesis, developments and metamorphoses, in IRMA Lectures in Math. Theor. Phys., volume 1, De Gruyter, Berlin Germany (2002), pp. 9-54 [math.QA/0201168].

[4] S. Waldmann, Poisson-Geometrie und Deformationsquantisierung, Springer, Berlin Germany (2007).

[5] C.-S. Chu and P.-M. Ho, Noncommutative open string and D-brane, Nucl. Phys. B 550 (1999) 151 [hep-th/9812219] [INSPIRE].

[6] V. Schomerus, D-branes and deformation quantization, JHEP 06 (1999) 030 [hep-th/9903205] [INSPIRE].

[7] L. Cornalba and R. Schiappa, Nonassociative star product deformations for D-brane world volumes in curved backgrounds, Commun. Math. Phys. 225 (2002) 33 [hep-th/0101219] [INSPIRE].

[8] M. Herbst, A. Kling and M. Kreuzer, Star products from open strings in curved backgrounds, JHEP 09 (2001) 014 [hep-th/0106159] [INSPIRE].

[9] M. Herbst, A. Kling and M. Kreuzer, Cyclicity of nonassociative products on D-branes, JHEP 03 (2004) 003 [hep-th/0312043] [INSPIRE].

[10] R. Blumenhagen and E. Plauschinn, Nonassociative gravity in string theory?, J. Phys. A 44 (2011) 015401 [arXiv:1010.1263] [InSPIRE].

[11] D. Lüst, T-duality and closed string non-commutative (doubled) geometry, JHEP 12 (2010) 084 [arXiv: 1010.1361] [INSPIRE].

[12] R. Blumenhagen, A. Deser, D. Lüst, E. Plauschinn and F. Rennecke, Non-geometric fluxes, asymmetric strings and nonassociative geometry, J. Phys. A 44 (2011) 385401 [arXiv:1106.0316] [INSPIRE].

[13] R. Jackiw, Three-cocycle in mathematics and physics, Phys. Rev. Lett. 54 (1985) 159 [INSPIRE].

[14] I. Bakas and D. Lüst, 3-cocycles, non-associative star-products and the magnetic paradigm of R-flux string vacua, JHEP 01 (2014) 171 [arXiv:1309.3172] [INSPIRE].

[15] C. Klimčík and T. Strobl, WZW-Poisson manifolds, J. Geom. Phys. 43 (2002) 341 [math.SG/0104189] [INSPIRE].

[16] P. Ševera and A. Weinstein, Poisson geometry with a 3-form background, Prog. Theor. Phys. Suppl. 144 (2001) 145 [math.SG/0107133] [INSPIRE]. 
[17] A. Alekseev, Y. Kosmann-Schwarzbach and E. Meinrenken, Quasi-Poisson manifolds, Can. J. Math. 54 (2002) 3.

[18] P. Ševera, Quantization of Poisson families and of twisted Poisson structures, Lett. Math. Phys. 63 (2003) 105 [math.QA/0205294].

[19] P. Aschieri, I. Baković, B. Jurčo and P. Schupp, Noncommutative gerbes and deformation quantization, J. Geom. Phys. 60 (2010) 1754 [hep-th/0206101] [INSPIRE].

[20] P. Bressler, A. Gorokhovsky, R. Nest and B. Tsygan, Deformation quantization of gerbes, Adv. Math. 214 (2007) 230 [math.QA/0512136].

[21] P. Bressler, A. Gorokhovsky, R. Nest and B. Tsygan, Deformations of algebroid stacks, Adv. Math. 226 (2011) 3018 [arXiv:0810.0030].

[22] F. Petalidou, On the geometric quantization of twisted Poisson manifolds, J. Math. Phys. 48 (2007) 083502 [arXiv:0704.2989].

[23] D. Mylonas, P. Schupp and R.J. Szabo, Non-geometric fluxes, quasi-Hopf twist deformations and nonassociative quantum mechanics, J. Math. Phys. 55 (2014) 122301 [arXiv: 1312.1621] [INSPIRE].

[24] D. Mylonas, P. Schupp and R.J. Szabo, Membrane $\sigma$-models and quantization of non-geometric flux backgrounds, JHEP 09 (2012) 012 [arXiv:1207.0926] [INSPIRE].

[25] V.G. Kupriyanov and D.V. Vassilevich, Star products made (somewhat) easier, Eur. Phys. J. C 58 (2008) 627 [arXiv:0806.4615] [inSPIRE].

[26] M. Bordemann, N. Neumaier and S. Waldmann, Homogeneous Fedosov star products on cotangent bundles I: Weyl and standard ordering with differential operator representation, Commun. Math. Phys. 198 (1998) 363 [q-alg/9707030] [inSPIRE].

[27] W. Behr and A. Sykora, Construction of gauge theories on curved noncommutative space-time, Nucl. Phys. B 698 (2004) 473 [hep-th/0309145] [INSPIRE].

[28] S. Gutt, An explicit *-product on the cotangent bundle of a Lie group, Lett. Math. Phys. 7 (1983) 249.

[29] G. Dito, Kontsevich star product on the dual of a Lie algebra, Lett. Math. Phys. 48 (1999) 307 [math.QA/9905080].

[30] N. Durov, S. Meljanac, A. Samsarov and Z. Skoda, A universal formula for representing Lie algebra generators as formal power series with coefficients in the Weyl algebra, J. Algebra 309 (2007) 318 [math. RT/0604096] [INSPIRE].

[31] S. Meljanac and M. Stojić, New realizations of Lie algebra kappa-deformed Euclidean space, Eur. Phys. J. C 47 (2006) 531 [hep-th/0605133] [INSPIRE].

[32] S. Meljanac and S. Krešić-Jurić, Generalized kappa-deformed spaces, star-products and their realizations, J. Phys. A 41 (2008) 235203 [arXiv:0804.3072] [INSPIRE].

[33] C. Chryssomalakos and E. Okon, Star product and invariant integration for Lie type noncommutative spacetimes, JHEP 08 (2007) 012 [arXiv:0705.3780] [INSPIRE].

[34] C. Guedes, D. Oriti and M. Raasakka, Quantization maps, algebra representation and non-commutative Fourier transform for Lie groups, J. Math. Phys. 54 (2013) 083508 [arXiv: 1301.7750] [INSPIRE].

[35] V.G. Kupriyanov and P. Vitale, Noncommutative $\mathbb{R}^{d}$ via closed star product, JHEP 08 (2015) 024 [arXiv: 1502.06544] [INSPIRE]. 
[36] M. Bojowald, S. Brahma, U. Buyukcam and T. Strobl, States in non-associative quantum mechanics: uncertainty relations and semiclassical evolution, JHEP 03 (2015) 093 [arXiv: 1411.3710] [INSPIRE].

[37] M. Penkava and P. Vanhaecke, Deformation quantization of polynomial Poisson algebras, J. Algebra 227 (2000) 365 [math.QA/9804022].

[38] G. Dito, The necessity of wheels in universal quantization formulas, Commun. Math. Phys. 338 (2015) 523 [INSPIRE].

[39] P. Schaller and T. Strobl, Poisson structure induced (topological) field theories, Mod. Phys. Lett. A 9 (1994) 3129 [hep-th/9405110] [INSPIRE].

[40] A.S. Cattaneo and G. Felder, A path integral approach to the Kontsevich quantization formula, Commun. Math. Phys. 212 (2000) 591 [math.QA/9902090] [INSPIRE].

[41] D.V. Vassilevich, Holographic duals to poisson $\sigma$-models and noncommutative quantum mechanics, Phys. Rev. D 87 (2013) 104011 [arXiv:1301.7029] [INSPIRE]. 